\title{
Simple and rapid separation of diverse neoagaro-oligosaccharides
}

\author{
Fudi Lin ${ }^{1}$, Yayan Huang ${ }^{1}$, Na Zhang ${ }^{1}$, Jing Ye ${ }^{1}$, Meitian Xiao ${ }^{1,2^{*}}$
}

${ }^{1}$ College of Chemical Engineering, Huaqiao University, Xiamen, Fujian, China

${ }^{2}$ Xiamen Engineering and Technological Research Center for Comprehensive Utilization of Marine Biological Resources, Xiamen, Fujian, China

*Corresponding author.

E-mail: mtxiao@hqu.edu.cn (MTX). 


\section{Abstract}

A rapid and simple method for obtaining pure and well-defined oligosaccharides was established by hydrolyzing agar with $\beta$-agarase from Vibrio natriegens. The conditions for enzymolysis were optimized as follows: temperature of $45^{\circ} \mathrm{C}$, $\mathrm{pH}$ of 8.5 , substrate concentration of $0.3 \%$, enzyme amount of $100 \mathrm{U} / \mathrm{g}$ and enzymolysis time of $20 \mathrm{~h}$. Neoagaro-oligosaccharides with different degree of polymerizations were gained by hydrolyzing agar with $\beta$-agarase at different enzymolysis time. After removing pigment by activated carbon and salts by dialyzing, the enzyme hydrolysis solution was separated with Bio-Gel P2 column chromatography. Neoagaro-oligosaccharides with different degree of polymerizations were acquired. By comparing with standard substances, along with further confirmation by FTIR, MS and NMR, structures of the purified neoagaro-oligosaccharides were identified as neoagarobiose, neoagaroteraose, neoagarohexaose, neoagarooctaose, neoagarodecaose and neoagarododecaose with purities more than $97.0 \%$, respectively. The present study established a method for rapid preparation of various monomers of neoagaro-oligosaccharides that may be of great significance for further study of bioactivities.

Keywords: Agar; Enzymatic hydrolysis; Neoagaro-oligosaccharides; Separation

\section{Introduction}

Agar, an important marine polysaccharide extracted from the cell walls of red algae, is a linear polymer containing $(1 \rightarrow 4)$-linked 3,6-anhydro- $\alpha$-L-galactose and $(1 \rightarrow 3)$-linked $\beta$-D-galactopyranose [1], and it is composed of agarose and agaropectin [2]. Agar is widely used in food, biological, and pharmaceutical industries [3]. Accumulating reports have indicated the oligosaccharides prepared from agar/agarose have diverse physiological functions such as antioxidant [4-8], anti-hyperlipidemia [9, 10], anti-inflammation [11-13], and whitening effect $[14,15]$ etc, which will expand its use in the food, cosmetic, and medical industries.

Generally, oligosaccharides from agar/agarose can be classified into agaro-oligosaccharides (AOS) [16-18] and neoagaro-oligosaccharides (NAOS) [19-21], the former are the hydrolysis products of acid or $\alpha$-agarase which cleaves $\alpha$ - $(1 \rightarrow$ 
3)-galactosidic bond of the polysaccharides, and the latter are hydrolysates of $\beta$-agarase which splits $\beta-(1 \rightarrow 4)$ bond. AOS and NAOS with different degree of polymerizations (DPs) have been reported to possess various bioactivities. A large amount of reports indicated that AOS have the beneficial effects of antioxidant [4-8], anti-obesity $[9,10]$, anti-inflammation [11-13], anti-cancer [22], and protect the intestine [9, 10, 22] etc. In recent years, NAOS mixtures as well as NOAS of signal DP have obtained increasing attention for its distinct physiological and biological activities. According to the reported prebiotic studies, the NAOS with DPs of 4-12 could increase the amount of lactobacilli and bifidobacteri in vivo, suggesting they had good probiotic effect [23]. Neoagarobiose (NA2), neoagaroteraose (NA4), and neoagarohexaose (NA6) were reported to have in vitro skin whitening and moisturizing effects, among them, NA4 was found to be a better whitening agent than the other two, whereas AOS did not exhibit the same activities[14, 15]. Moreover, NA4 displayed better ability to scavenge hydroxyl radicals compared with NA2, NA6, and neoagarooctaose (NA8), meanwhile, the scavenging activities on hydroxyl radicals was not found for agarotriose and agarobiose. Additionally, NA4 was proved to inhibit inflammation in LPS-stimulated macrophages through suppression of MAPK and NF- $\kappa B$ pathways [24, 25].

Notably, these studies mainly focused on the activities of NAOS mixture, NA2, NA4, and NA6, while few reports investigated the bioactivities of NAOS with higher DPs such as NA8, neoagarodecaose (NA10), and neoagarododecaose (NA12), etc. The reasons may be attributed to the difficulty of obtaining NAOS with higher DPs and the complexity of purification of them. Many efforts have been devoted to obtain NAOS. $\beta$-Agarases from marine bacterium Janthinobacterium sp. SY12, Vibrio sp. Strain JT0107, Agarivorans albus YKW-34 and Agarivorans albus OAY02 were employed to degrade agarose to give NA2 and NA4 [26-28]. And $\beta$-agarase obtained from Microbulbifer sp. Q7, marine Alteromonas sp. SY37-12, Pseudoalteromonas sp. CY24, marine Agarivorans sp. LQ48 and Pseudoalteromonas sp. AG4 could hydrolyze $\beta-1,4$-glycosidic linkages of agarose/agar to gain NA4 and NA6 [29-33]. $\beta$-Agarases isolated from Stenotrophomonas sp. NTa and Agarivorans sp. JA-1 in Bacillus subtilis degraded agarose/agar to NA2, NA4 and NA6 as the predominant products [34, 35]. 
Agarivorans albus OAY02 could secrete two $\beta$-agarases, among them, one $\beta$-agarase could cleave agarose into NA2 and NA4, the other $\beta$-agarase made agarose become NA2, NA4, NA6 and NA8 [36]. And $\beta$-agarase from marine Pseudoalteromonas sp. CY24 could degrade agarose to NA8 and NA10 [37]. $\beta$-Agarase from marine bacteria can degrade agarose to NAOS with different DPs, however, up to now it has not been reported that $\beta$-agarase could degrade agarose/agar to obtain NAOS with desired DPs at different enzymolysis time.

Developing methods for rapid separation and purification of NAOS is also very important for obtaining purified NAOS. Size-sieving based on gel-permeation chromatography (GPC) and high performance anion exchange chromatography (HPAEC) are commonly used methods for the separation of polysaccharides and oligosaccharides [6, 38-41]. Toyopearl HW-40S was used to purify NA4[38]. It was reported that NAOS with DP of 2, 4, and 6 could be separated and purified by Bio-Gel P2 [40, 41] and NA4, NA6, NA8, NA10 and NA12 could be separated by two chromatography steps of consecutive Bio-Gel P-6 chromatography [40]. Sephadex G-10 combined with G-25 was employed to purify AOS $[6,39]$. The SEC-HPLC and $\mathrm{NH}_{2}-\mathrm{HPLC}$ systems were used to isolate and purify NAOS and AOS [42]. A HPAEC system equipped with a semi-preparative CarboPac ${ }^{\mathrm{TM}}$ PA100 column was applied to prepare NAOS and AOS from DP 2 to DP 22 with product yield and purity no more than $17.2 \%$ and $77.7 \%$, respectively [43]. However, these methods are a little bit complicated, time-consuming, and instrument-depending, and the product yield and purity of obtained oligosaccharides are not high enough for further study. Therefore, to deeply understand the bioactivities and the mechanism of NOAS, it is urgent to develop a simple and rapid method for the preparation of NAOS with various DPs.

In the present study, a simple method of obtaining NAOS with desired DPs was established by regulating the enzymolysis time of $\beta$-agarases. And a gel filtration chromatography was developed for the rapid preparation of each NAOS with different DPs in high quality and quantity, making it possible to the further studies on their bioactivities. 


\section{Materials and methods}

\section{Strains and reagents}

NA2, NA4, NA6, NA8, NA10 and NA12 used as standards were purchased from Qingdao bozhihui Biological Technology Co. Ltd (Qingdao, China). Acetonitrile were gained from Sigma-Aldrich (St. Louis, MO, USA). All the other reagents were commercially available and of analytical grade.

The culture and fermentation condition of Vibrio natriegens was the same as what was explored in our previous work[44]. The obtained $\beta$-agarase was purified from the fermentation liquor with the combination of ammonium sulfate salting-out, dialysis, ion exchange chromatography and gel-filtration. The enzymatic activity of the purified $\beta$-agarase reached $103 \mathrm{U} / \mathrm{mL}$, which was applied in this work for the later experiment.

\section{Optimization of enzymatic hydrolysis condition}

The enzymatic hydrolysis condition including reaction temperature $(30,35,40,45$, 50,55 and $\left.60^{\circ} \mathrm{C}\right), \mathrm{pH}(6.0,6.5,7.0,7.5,8.0,8.5$ and 9.0), reaction time $(1,5,10,15,20$, 25 and $30 \mathrm{~h})$, substrate concentration $(0.1,0.2,0.3,0.4,0.5,0.6$ and $0.7 \%)$ and enzyme amounts $(20,40,60,80,100,120$ and $140 \mathrm{U} / \mathrm{g})$ were optimized. All hydrolysis reactions were conducted in triplicate.

\section{Preparation of NAOS products with different enzymolysis time}

$3 \%$ Agar solution was completely dissolved in $1 \mathrm{~L}$ of $0.1 \mathrm{M}$ Tris- $\mathrm{HCl}$ solution by heating and then cooled to $45{ }^{\circ} \mathrm{C}$. Subsequently, the solution was treated with the $\beta$-agarase for $4 \mathrm{~h}, 6 \mathrm{~h}, 8 \mathrm{~h}, 10 \mathrm{~h}$ and $12 \mathrm{~h}$ to obtain products $\mathrm{A}, \mathrm{B}, \mathrm{C}, \mathrm{D}$ and $\mathrm{E}$, respectively. After inactivation with boiling water bath for 15 minutes and centrifugation for $30 \mathrm{~min}(12,000 \times \mathrm{g})$, the insoluble agar was removed and the NAOS in supernatant was filtered with a $0.22 \mu \mathrm{m}$ membrane (Millipore, Cork, Ireland). The crude products of different enzymolysis time were finally obtained by lyophilization and stored at $-20{ }^{\circ} \mathrm{C}$ for further use. 


\section{Analysis of NAOS products by HPLC-ELSD}

The NAOS products were analyzed by HPLC-ELSD system which consisted of a Waters e2695 HPLC system (Waters, Milford, MA, USA) equipped with an evaporative light scattering detector (Waters 2424, USA). Separation was performed on an Asahipak $\mathrm{NH}_{2} \mathrm{P}-504 \mathrm{E}$ multi-mode analytical column $(250 \mathrm{~mm} \times 4.6 \mathrm{~mm}, 5 \mu \mathrm{m})$ with the column temperature of $30{ }^{\circ} \mathrm{C}$. Isocratic elution was conducted with acetonitrile-water (65:35) as the mobile phase with a flow rate of $1 \mathrm{~mL} / \mathrm{min}$. The injection volume was set at $10 \mu \mathrm{L}$ and the detector nebulizer temperature was $75^{\circ} \mathrm{C}$.

\section{Rapid purification of NAOS}

For the purification of NAOS, $1 \mathrm{~g}$ crude NAOS product powder was resuspended in $200 \mathrm{~mL}$ distilled water, followed by addition of $10 \mathrm{~g}$ activated carbon. The mixture was stirred for $2 \mathrm{~h}$ and then NAOS were washed out by $30 \%$ ethanol solution and ethanol was removed by rotary evaporator. Then the left was freeze dried and detected.

GPC was applied to separate the NAOS. $250 \mathrm{mg}$ of products A was resuspended in 1 $\mathrm{mL}$ of $\mathrm{NH}_{4} \mathrm{HCO}_{3}(0.1 \mathrm{M})$, and the solutions were loaded onto the Bio-Gel P2 column (18 $\times 150 \mathrm{~cm}$, Bio-Rad Laboratories, Hercules, CA, USA), respectively. $\mathrm{NH}_{4} \mathrm{HCO}_{3}$ was used as eluent at a flow rate of $0.4 \mathrm{~mL} / \mathrm{min}$ and fractions of $4 \mathrm{~mL}$ each were collected. Then the collected fractions were detected by TLC on a Silica Gel 60 plate (Merck, Darm-stadt, Germany) developed with a solvent of isopropanol/water/ammonium hydroxide (30:15:2, $\mathrm{v} / \mathrm{v} / \mathrm{v}$ ). After being sprayed by anisaldehyde and heated for $10 \mathrm{~min}$, the spot of the products could be visualized.

\section{Identification of NAOS}

The structure of isolated NAOS was elucidated by FTIR and the molecular mass was confirmed by ESI-TOF-MS analysis. The FTIR spectra of KBr pellets of the NAOS after drying at $105{ }^{\circ} \mathrm{C}$ for $2 \mathrm{~h}$ were recorded on the FTIR (FTIR-84, Shimadzu, Japan) spectrophotometer. Scans were performed over the range of $4000-400 \mathrm{~cm}^{-1}$ with a resolution of $4 \mathrm{~cm}^{-1}$ for 32 times. ESI-TOF-MS analysis was carried out on a Q Exactive Hybrid Quadrupole Orbitrap mass spectrometer (Thermo, Bremen, Germany) coupled 
with an ESI source in positive ion mode. Recorded mass range was $m / z 200-2000$. $1 \mathrm{H}-\mathrm{NMR}$ and $13 \mathrm{C}-\mathrm{NMR}$ spectra were measured in $\mathrm{D}_{2} \mathrm{O}$ on a Avance 500 spectrometer (Bruker, Avance III, Switzerland, $500 \mathrm{MHz} \mathrm{1H,} 125 \mathrm{MHz}$ 13C) at room temperature and $\mathrm{C}_{3} \mathrm{D}_{6} \mathrm{O}$ was added as an internal standard with the chemical shifts were reported in $\delta=$ $2.05 \mathrm{ppm}$ for $1 \mathrm{H}-\mathrm{NMR}, \delta=29.84 \mathrm{ppm}$ for $13 \mathrm{C}-\mathrm{NMR}$.

\section{Results and discussion}

\section{Optimization of hydrolysis parameters by single factor}

\section{experiments}

Single factor experiments were carried out to optimize the enzymatic hydrolysis process. Fig. 1a displayed the influence of temperature on the yield of reducing sugar. Temperature is an important factor in the process of enzymatic hydrolysis. Within a certain temperature range, the raise of temperature is beneficial to enzymatic reaction. As shown in Fig. 1a, the yield of reducing sugar was increased with the elevation of temperature and it reached $45.2 \%$ at the temperature of $45{ }^{\circ} \mathrm{C}$, after that the yield was decreased significantly with the raise of temperature. Therefore, the temperature was set at $45{ }^{\circ} \mathrm{C}$. The $\mathrm{pH}$ is another vital parameter in the enzymatic hydrolysis process, because enzyme activity is greatly affected by $\mathrm{pH}$ value. Generally, an enzyme can maintain high enzyme activity under suitable pH. As known in Fig. 1b, the yield of reducing sugar was increased with the increase of $\mathrm{pH}$ from 6.0 to 8.5 , the largest yield of reducing sugar was $42.7 \%$ at $\mathrm{pH}$ 8.5. Then the yield was decreased, which may be attributed to the increase of $\mathrm{pH}$ on the inhibition of the $\beta$-agarase activity. As for the substrate concentration, low substrate concentration leads to low enzyme utilization, however, too high the substrate concentration will hinder the diffusion of molecules and reduce the enzymatic reaction rate. As showed in Fig. 1c, when the substrate concentration was set at $0.3 \%$, the yield of reducing sugar reached the highest of $43.3 \%$. In addition, not only enzyme amount but also enzymolysis time has prominent effects on the yield of reducing sugar $[45,46]$. The enzyme amounts were also investigated in the present study and the adding amount at $100 \mathrm{U} / \mathrm{g}$ turned out to be optimal with the reducing sugar yield of $42.4 \%$, and the yield of 
reducing sugar had remained almost stable after the enzyme amount added was more than $100 \mathrm{U} / \mathrm{g}$. Fig. 1e indicated that the yield of reducing sugar increased with the extension of enzymolysis time before the time of $20 \mathrm{~h}$. When the enzymolysis time was $20 \mathrm{~h}$, reducing sugar yield reached the highest at $43.3 \%$, and the generation of reducing sugar didn't appear to increase over $20 \mathrm{~h}$ of enzymolysis time. Therefore, the optimized conditions were optimized as follows: the temperature of $45{ }^{\circ} \mathrm{C}$, the $\mathrm{pH}$ of 8.5 , the substrate concentration of $0.3 \%$, the enzyme amount of $100 \mathrm{U} / \mathrm{g}$ and the enzymolysis time of $20 \mathrm{~h}$.

\section{Fig. 1 Effects of enzymolysis condition on reducing sugar yield}

\section{Preparation of NAOS with different DPs}

The $\beta$-agarase obtained by the different strains would degrade agar to gain the NAOS with different DPs $[26,30,34,35]$. Specifically, the regular changes of NAOS with different DPs at different enzymolysis time were found in the present study (Fig.2), which was not mentioned in the previous reports. The enzymolysis products were determined with HPLC-ELSD by comparing with standard substances and the content of each oligosaccharide was calculated from the regression equations, as shown in Fig.2 and table 1 and table 2, and the enzymolysis product was analyzed to be composed of NA2, NA4, NA6, NA8, NA10 and NA12 with the yield of 5.0\%, 38.9\%, 18.1\%, 16.8\%, 13.8\% and $2.5 \%$ respectively after the agar was hydrolyzed by $\beta$-agarase for $4 \mathrm{~h}$. When the hydrolysis time was 6 h, NA12 was completely hydrolyzed and the product obtained was NA2, NA4, NA6, NA8, and NA10, the yield were $6.0 \%, 52.7 \%, 15.2 \%, 13.7 \%$, and $11 \%$, respectively. As the hydrolysis time prolonged to $8 \mathrm{~h}$, NA10 was not detected in the product and the yield for NA2, NA4, NA6 and NA8 were 9.3\%, 59.5\%, 13.7\% and $11.7 \%$, respectively. With the extension of the hydrolysis time, the DPs of NAOS obtained were getting smaller and smaller. When the hydrolysis time was $10 \mathrm{~h}$, the product was consisted of NA2, NA4, and NA6 with the yield of $13.8 \%, 68.4 \%$ and $9.6 \%$ separately. And the hydrolysis time reached $12 \mathrm{~h}$, only NA4 and NA2 were left in the product with the yield of $21.6 \%$ and $71.1 \%$, and the product components were no longer changed with the extension of time.

Fig. 2 Anlysis of enzymatic hydrolysates with different enzymolysis time, S-NAOS 
standards, 1-NA2, 2-NA4, 3-NA6, 4-NA8, 5-NA10, 6- NA12; A 4 h, B 6 h, C 8 h, D 10 h, E 12 h

Table. 1 The regression equations of NAOS

\begin{tabular}{ccc}
\hline Oligosaccharide & Equation & $\mathrm{R}^{2}$ \\
\hline NA2 & $\mathrm{y}=6.29305 \mathrm{x}-2.21022$ & 0.992 \\
NA4 & $\mathrm{y}=6.05888 \mathrm{x}-3.18131$ & 0.996 \\
NA6 & $\mathrm{y}=5.54881 \mathrm{x}-3.71928$ & 0.997 \\
NA8 & $\mathrm{y}=4.8549 \mathrm{x}-4.42057$ & 0.996 \\
NA10 & $\mathrm{y}=4.48493 \mathrm{x}-3.88547$ & 0.994 \\
NA12 & $\mathrm{y}=4.25971 \mathrm{x}-3.75222$ & 0.992 \\
\hline
\end{tabular}

$\mathrm{x}$ : the concentration of NAOS $(\mathrm{mg} / \mathrm{ml}), \mathrm{y}$ : peak area $\times 10^{-6}$.

Table. 2 The percentage of monomers at different hydrolysis time

\begin{tabular}{cccccc}
\hline & A & B & C & D & E \\
\hline NA2 (\%) & 5 & 6 & 9.3 & 13.8 & 21.6 \\
NA4 (\%) & 38.9 & 52.7 & 59.5 & 68.4 & 71.1 \\
NA6 (\%) & 18.1 & 15.2 & 13.7 & 9.6 & 0 \\
NA8 (\%) & 16.8 & 13.7 & 11.7 & 0 & 0 \\
NA10 (\%) & 13.8 & 11 & 0 & 0 & 0 \\
NA12 (\%) & 2.5 & 0 & 0 & 0 & 0 \\
\hline
\end{tabular}

At present, there are mounting reports on the preparation of NAOS by enzymatic method and agarase is mainly derived from the secretion of marine bacteria. Frequently $\beta$-agarase is found to degrade agar to generate NA2, NA4, or NA6 as the main products. The DagA secreted $\beta$-agarase which could hydrolyze agar to gain NA2, NA4 and NA6 [47]. And it was reported $\beta$-agarase from Stenotrophomonas sp. NTa degraded agar only obtained NA2, NA4 and NA6 as the predominant products and a small amount of 3,6-anhydro- $\alpha$-L-galactose, and notably the products did not change with the change of the hydrolysis time [35]. At the same time, the composition of the agarolytic did not changed over time by some $\beta$-agarase, which included four even-numbered NAOS with DP of 2-8, and the amount of NA4 was more than others [25]. However, these methods 
found in literature were used in preparing NAOs mainly with DP no more than eight [25, $35,47]$. Interestingly, we found in our study that the DPs of the NAOS reduced regularly with the enzymolysis time increased every two hours, and the final product was composed of NA4 and NA2. Therefore, desired NOAS with different DPs could be obtained by controlling the hydrolysis time, which was beneficial to the further studies of NAOS

\section{Rapid isolation of NAOS}

Separation and purification of NAOS was carried out using a Bio-Gel P2 column and detected by TLC, and the result was shown in Fig. 3. For product A, fractions of 18 to 22 were NA12 with the yield of $3.2 \%$, fraction 24 to fraction 27 were NA10 with the yield of $4.2 \%$, fraction 30 to fraction 35 was NA8 with the yield of $7.5 \%$, fraction 37 to fraction 40 was NA6 with the yield of $10.2 \%$, fraction 44 to fraction 56 was NA4 with the yield of $35.8 \%$, fraction 61 to fraction 64 was NA2 and the yield was $23.2 \%$, respectively. After detected by HPLC-ELSD, the purity of NA2, NA4, NA6, NA8, NA10 and NA12 were 99.3\%, 98.9\%, 98.0\%, 97.6\%, 97.3\% and 97.4\%, respectively (Fig.4).

GPC and HPAEC are commonly used method for separation and preparation of NAOS and Bio-Gel P-2 and Bio-Gel P-6 are frequently applied to purify NAOS [6, 38-43]. However, these two separation media are usually jointly used to prepare NAOS with diverse DPs. Noticeably, in the present study, one chromatography step of Bio-Gel P-2 column chromatography could be developed to obtain NAOS of DP2-12 with purities more than $97 \%$, suggesting it is a simple and rapid method for the preparation of NAOS.

Fig. 3 TLC analysis of purified NAOS. The ladder of NAOS with different DP (2-12); S: NAOS standards; Fractions 18-22: NA 12, Fractions 24-27: NA 10, Fractions 30-35: NA8, Fractions 37-40: NA 6, Fractions 44-56: NA4, Fractions 61-64: NA2 Fig. 4 High liquid chromatograms of purified products

\section{Structure and molecular confirmation of NAOS}

The structures and the molecular mass of the purified NAOS were confirmed by FTIR and MS analysis. Fig.5 demonstrated the results of the FTIR analyses. In all the six 
obtained oligosaccharides, the disappearance of absorption band around $1260 \mathrm{~cm}^{-1}$ indicated the elimination of sulfate group in the degradation process. There was a broad absorption band around $3400 \mathrm{~cm}^{-1}$, which may be assigned to hydroxyl group. The region around $2950 \mathrm{~cm}^{-1}$ and $2900 \mathrm{~cm}^{-1}$ were assigned to $\mathrm{C}-\mathrm{H}$. The band around $1640 \mathrm{~cm}^{-1}$ suggested the existence of C-C sugar ring. The fingerprint region, including many FTIR absorptions of specific characteristic bonds, was a region of lower wave numbers. There was absorption band around $1159 \mathrm{~cm}^{-1}$ was the stretch vibration of C-O within C-O-H. Absorption bands appeared at $1072 \mathrm{~cm}^{-1}$, indicating the presence of $\mathrm{C}-\mathrm{O}$ within $\mathrm{C}-\mathrm{O}-\mathrm{C}$ bond. A well-defined peak was shown at about $930 \mathrm{~cm}^{-1}$ corresponding to 3 , 6-anydro-D-galactose.

\section{Fig. 5 FTIR spectrum of NAOS}

The ESI-TOF-MS analysis results were displayed in on table 3, confirming the purified oligosaccharides were NA2, NA4, NA6, NA8, NA10 and NA12, respectively. The NA4 was shown in Fig. 6 and other monomers were in S1 Fig.

\section{Fig. 6 The ESIMS spectra of NA4}

Table 3 The ESI-TOF-MS analysis of the purified products

\begin{tabular}{ccc}
\hline purified products & $\begin{array}{c}\text { MS signals }(\mathrm{m} / \mathrm{z}) \\
{[\mathrm{M}+\mathrm{Na}]^{+}}\end{array}$ & $\begin{array}{c}\text { Calculated molecular } \\
\text { weights }\end{array}$ \\
\hline NA2 & 347.0949 & 347.0954 \\
NA4 & 653.1886 & 653.1905 \\
NA6 & 959.2866 & 959.2856 \\
NA8 & 1265.3752 & 1265.3807 \\
NA10 & 1571.4752 & 1571.4757 \\
NA12 & 1877.5751 & 1877.5708 \\
\hline
\end{tabular}

The structural information of six monomers was demonstrated by ${ }^{1} \mathrm{H}-\mathrm{NMR}$ and ${ }^{13} \mathrm{C}-\mathrm{NMR}$ spectroscopy. Assignments of ${ }^{1} \mathrm{H}-\mathrm{NMR}$ and ${ }^{13} \mathrm{C}-\mathrm{NMR}$ spectroscopy were built the close similarity with literature values, and the interpretation of these signals was shown in Fig. 7 and table $4[1,41,42]$. It indicated twelve particular major anomeric carbon signals ( $\mathrm{G}$ and $\mathrm{A}$ ), which was anticipated that the NA2 was the major repeat unit, 
and the signals (Gnr and Anr) were the residues towards the nonreducing end of the NAOS. Resonances at about 96.6 and $92.6 \mathrm{ppm}$ were characteristic of $\beta$ and $\alpha$ anomeric form of galactose residues at the reducing end of the NAOS[40, 48], respectively, and the NA4 of ${ }^{13} \mathrm{C}-\mathrm{NMR}$ was showed on Fig. 8, and ${ }^{1} \mathrm{H}-\mathrm{NMR}$ and ${ }^{13} \mathrm{C}-\mathrm{NMR}$ of other monomers were in S2 Fig and S3 Fig, respectively.

Fig. $7{ }^{1} \mathrm{H}$ NMR spectra of NA4, Peak labels: A, 3,6 anhydrogalactose; G, galactose; $\mathrm{nr}$ and $\boldsymbol{r}$ refer to the non-reducing and reducing ends; $\alpha, \beta$ refer to positions of protons on reducing ends; numbers from 1 to 6 refer to place of protons

Fig. $8^{13} \mathrm{C}$ NMR spectra of NA4

Table 4 Chemical shift assignments for ${ }^{13} \mathrm{C}-\mathrm{NMR}$ spectra of NAOS

\begin{tabular}{ccccccc}
\hline Unit & C1 & C2 & C3 & C4 & C5 & C6 \\
\hline Gnr & 102.2 & 69.9 & 82.0 & 67.8 & 75.1 & 61.3 \\
Gr $\beta$ & 96.6 & 69.9 & 82.5 & 68.6 & 75.1 & 61.3 \\
Gr $\alpha$ & 92.6 & 67.8 & 79.9 & 69.2 & 69.9 & 61.4 \\
Anr & 98.3 & 69.4 & 80.8 & 69.6 & 77.3 & 68.7 \\
Ar $\alpha$ & 98.1 & 69.5 & 79.9 & 77.3 & 75.3 & 69.0 \\
\hline
\end{tabular}

\section{Conclusions}

In summary, the present study has developed a feasible approaches for the preparation of desired NAOS with different DPs by regulating the enzymolysis time of $\beta$-agarase. Furthermore, the NAOS of diverse DPs were rapidly and simply prepared by the Bio-gel P2 column chromatography with purities higher than $97 \%$ for further evaluating their bioactivity potentials.

\section{Acknowledgment}

This work was supported by Subsidized Project for Postgraduates' Innovative Fund in Scientific Research of Huaqiao University; and the Public Science and Technology Research Funs Projects of Ocean [grant numbers 20130515-2, 201505026-5].

\section{Conflict of interest statement}

The authors declared no conflict of interest. 


\section{References}

316 1. Hamer GK, Bhattacharjee SS, Yaphe W. Analysis of the enzymlc hydmlysls products of agahose by

317 C-NMR. Carbohydrate research. 1977;54(1):C7-C10.

318 2. Jensen A. Present and future needs for algae and algal products. Hydrobiologia. 1993;260/261:15-23.

319 3. Chi WJ, Chang YK, Hong SK. Agar degradation by microorganisms and agar-degrading enzymes.

320 Applied microbiology and biotechnology. 2012;94(4):917-30. doi: 10.1007/s00253-012-4023-2. PubMed 321 PMID: 22526785.

322 4. Chen HM, Zheng L, Yan XJ. The preparation and bioactivity research of agaro-oligosaccharides.

323 Food Technology and Biotechnology. 2005;43(1):29-36.

324 5. Chen HM, Yan XJ. Antioxidant activities of agaro-oligosaccharides with different degrees of 325 polymerization in cell-based system. Biochimica et biophysica acta. 2005;1722(1):103-11. doi: 10.1016/j.bbagen.2004.11.016. PubMed PMID: 15716131.

327 6. Wu SC, Wen TN, Pan CL. Algal-oligosaccharide-lysates prepared by two bacterial agarases stepwise hydrolyzed and their anti-oxidative properties. Fisheries Science. 2005;71:1149-59.

7. Chen H, Yan X, Zhu P, Lin J. Antioxidant activity and hepatoprotective potential of agaro-oligosaccharides in vitro and in vivo. Nutrition journal. 2006;5:31-42. doi: 10.1186/1475-2891-5-31. PubMed PMID: 17140450; PubMed Central PMCID: PMC1698930.

8. Kang OL, Ghani M, Hassan O, Rahmati S, Ramli N. Novel agaro-oligosaccharide production through enzymatic hydrolysis: Physicochemical properties and antioxidant activities. Food Hydrocolloids. 2014;42:304-8. doi: 10.1016/j.foodhyd.2014.04.031.

9. Higashimura Y, Naito Y, Takagi T, Uchiyama K, Mizushima K, Ushiroda C, et al. Protective effect of agaro-oligosaccharides on gut dysbiosis and colon tumorigenesis in high-fat diet-fed mice. American Journal of Physiology-Gastrointestinal and Liver Physiology. 2016;310(6):G367-G75. doi: 10.1152/ajpgi.00324.2015.-High-fat.

10. Higashimura Y, Baba Y, Inoue R, Takagi T, Mizushima K, ., Ohnogi H, et al. Agaro-oligosaccharides regulate gut microbiota and adipose tissue accumulation in mice. Journal of Nutritional Science and Vitaminology. 2017;63(4):269-76.

11. Enoki T, Tanabe M, Shimomura M, Ohnogi H. Induction mechanism of heme oxygenase-1 and anti-inflammatory activity by agaro-oligosaccharides. Nippon Shokuhin Kogyo Gakkaishi. 2010;57(4):157-62.

12. Enoki T, Tominaga T, Takashima F, Ohnogi H, Sagawa H, Kato I. Anti-tumor-promoting activities of agaro-oligosaccharides on two-stage mouse skin carcinogenesis. Biological \& Pharmaceutical Bulletin. 2012;35(7):1145-9.

348 13. Higashimura Y, Naito Y, Takagi T, Tanimura Y, Mizushima K, Harusato A, et al. Preventive effect of agaro-oligosaccharides on non-steroidal anti-inflammatory drug-induced small intestinal injury in mice. Journal of gastroenterology and hepatology. 2014;29(2):310-7. doi: 10.1111/jgh.12373. PubMed PMID: 23980531.

14. Kobayashi R, Takisada M, Suzuki T, Kirimuraab K, Usamiab S. Neoagarobiose as a novel moisturizer 10.1271/bbb.61.162. 
2017;15(10):321-30. doi: 10.3390/md15100321. PubMed PMID: 29053566; PubMed Central PMCID: PMC5666429.

16. Potin P, Richard C, Rochas C, Kloareg B. Purification and characterization of the $\alpha$-agarase from Alteromonas agarlyticus (Cataldi) comb. nov., strain GJ1B. European Journal of Biochemistry 1993;214:559-607.

17. Hassairi I, Amar RB, Nonus M, Gupta BB. Production and separation of $\alpha$-agarase from Altermonas agarlyticus strain GJ1B. Bioresource technology. 2001;79(1):47-51.

18. Ohta Y, Hatada Y, Miyazaki M, Nogi Y, Ito S, Horikoshi K. Purification and characterization of a novel alpha-agarase from a Thalassomonas sp. Current microbiology. 2005;50(4):212-6. doi: 10.1007/s00284-004-4435-z. PubMed PMID: 15902469.

19. Kirimura K, Masuda N, Iwasaki Y, Nakagawa H, Kobayashi R, Usami S. Purification and characterization of a novel $\beta$-agarase from an alkalophilic bacterium, Alteromonas sp. E-1. Journal of bioscience and bioengineering. 1999;87:436-41.

20. Kang NY, Choi YL, Cho YS, Kim BK, Jeon BS, Cha JY, et al. Cloning, expression and characterization of a $\beta$-agarase gene from a marine bacterium, Pseudomonas sp. SK38. Biotechnology Letters. 2003;25:1165-70.

21. Fu W, Han B, Duan D, Liu W, Wang C. Purification and characterization of agarases from a marine bacterium Vibrio sp. F-6. Journal of industrial microbiology \& biotechnology. 2008;35(8):915-22. doi: 10.1007/s10295-008-0365-2. PubMed PMID: 18478285.

22. Bhattarai Y, Kashyap PC. Agaro-oligosaccharides: a new frontier in the fight against colon cancer? American Journal of Physiology-Gastrointestinal and Liver Physiology ～2016;310(6):ajpgi.00049.2016. doi: 10.1152/ajpgi.00049.2016. PubMed PMID: 26867562; PubMed Central PMCID: PMC4796294.

23. Hu B, Gong Q, Wang Y, Ma Y, Li J, Yu W. Prebiotic effects of neoagaro-oligosaccharides prepared by enzymatic hydrolysis of agarose. Anaerobe. 2006;12(5-6):260-6. doi: 10.1016/j.anaerobe.2006.07.005. PubMed PMID: 16973391.

24. Wang W, Liu P, Hao C, Wu L, Wan W, Mao X. Neoagaro-oligosaccharide monomers inhibit inflammation in LPS-stimulated macrophages through suppression of MAPK and NF-kappaB pathways. Scientific reports. 2017;7:44252. doi: 10.1038/srep44252. PubMed PMID: 28266652; PubMed Central PMCID: PMC5339798.

25. Xu XQ, Su BM, Xie JS, Li RK, Yang J, Lin J, et al. Preparation of bioactive neoagaroligosaccharides through hydrolysis of Gracilaria lemaneiformis agar: A comparative study. Food Chemistry. 2018;240:330-7. doi: 10.1016/j.foodchem.2017.07.036. PubMed PMID: 28946280.

26. Shi YL, Lu XZ, Yu WG. A new $\beta$-agarase from marine bacterium Janthinobacterium sp. SY12. World Journal of Microbiology and Biotechnology. 2008;24(11):2659-64. doi: 10.1007/s11274-008-9792-5.

27. Fu XT, Lin H, Kim SM. Purification and characterization of a novel beta-agarase, AgaA34, from Agarivorans albus YKW-34. Applied microbiology and biotechnology. 2008;78(2):265-73. doi: 10.1007/s00253-007-1303-3. PubMed PMID: 18071641.

28. Sugano Y, Terada I, Arita M, Noma M, Matsumoto T. Purification and characterization of a new agarase from a marine bacterium, Vibrio sp. strain JT0107. Applied and environmental microbiology. 1993; 59(5):1549-54.

29. Su Q, Jin T, Yu Y, Yang M, Mou H, Li L. Extracellular expression of a novel beta-agarase from Microbulbifer sp. Q7, isolated from the gut of sea cucumber. AMB Express. 2017;7(1):220. doi: 10.1186/s13568-017-0525-8. PubMed PMID: 29260432; PubMed Central PMCID: PMC5736513. 
30. Wang J, Mou H, Jiang X, Guan H. Characterization of a novel beta-agarase from marine Alteromonas sp. SY37-12 and its degrading products. Applied microbiology and biotechnology. 2006;71(6):833-9. doi: 10.1007/s00253-005-0207-3. PubMed PMID: 16317543.

31. Lu X, Chu Y, Wu Q, Gu Y, Han F, Yu W. Cloning, expression and characterization of a new agarase-encoding gene from marine Pseudoalteromonas sp. Biotechnology Letters. 2009;31(10):1565-70. doi: 10.1007/s10529-009-0042-1. PubMed PMID: 19504047.

32. Long $\mathrm{M}, \mathrm{Yu} \mathrm{Z}, \mathrm{Xu} \mathrm{X}$. A novel beta-agarase with high $\mathrm{pH}$ stability from marine Agarivorans sp. LQ48. Marine biotechnology. 2010;12(1):62-9. doi: 10.1007/s10126-009-9200-7. PubMed PMID: 19484308.

33. Oh C, Nikapitiya C, Lee Y, Whang I, Kim SJ, Kang DH, et al. Cloning, purification and biochemical characterization of beta agarase from the marine bacterium Pseudoalteromonas sp. AG4. Journal of industrial microbiology \& biotechnology. 2010;37(5):483-94. doi: 10.1007/s10295-010-0694-9. PubMed PMID: 20213114.

34. Lee DG, Jang MK, Lee OH, Kim NY, Ju SA, Lee SH. Over-production of a glycoside hydrolase family 50 beta-agarase from Agarivorans sp. JA-1 in Bacillus subtilis and the whitening effect of its product. Biotechnology Letters. 2008;30(5):911-8. doi: 10.1007/s10529-008-9634-4. PubMed PMID: 18189120

35. Zhu Y, Zhao R, Xiao A, Li L, Jiang Z, Chen F, et al. Characterization of an alkaline beta-agarase from Stenotrophomonas sp. NTa and the enzymatic hydrolysates. International journal of biological macromolecules. 2016;86:525-34. doi: 10.1016/j.ijbiomac.2016.01.106. PubMed PMID: 26836616.

36. Yang M, Mao X, Liu N, Qiu Y, Xue C. Purification and characterization of two agarases from Agarivorans albus OAY02. Process Biochemistry. 2014;49(5):905-12. doi: 10.1016/j.procbio.2014.02.015. 37. Ma C, Lu X, Shi C, Li J, Gu Y, Ma Y, et al. Molecular cloning and characterization of a novel beta-agarase, AgaB, from marine Pseudoalteromonas sp. CY24. Journal of Biological Chemistry. 2007;282(6):3747-54. doi: 10.1074/jbc.M607888200. PubMed PMID: 17166842.

38. Osumi Y, Kawai M, Amano H, Noda H. Purification and structure of oligosaccharides from porphyran degradated by enzymes from Arthrobacter sp. S-22. Nippon Suisan Gakkaishi. 1998;64:88-97.

39. Chen HM, Zheng L, Lin W, Yan XJ. Product monitoring and quantitation of oligosaccharides composition in agar hydrolysates by precolumn labeling HPLC. Talanta. 2004;64(3):773-7. doi: 10.1016/j.talanta.2004.04.002. PubMed PMID: 18969671.

40. Li J, Han F, Lu X, Fu X, Ma C, Chu Y, et al. A simple method of preparing diverse neoagaro-oligosaccharides with beta-agarase. Carbohydrate research. 2007;342(8):1030-3. doi: 10.1016/j.carres.2007.02.008. PubMed PMID: 17359946.

41. Morrice LM, McLean MW, Long WF, Williamson FB. $\beta$-Agarases I and II from Pseudomonas atlantica. European Journal of Biochemistry. 1983;137:149-54.

42. Kazlowski B, Pan CL, Ko YT. Separation and quantification of neoagaro- and agaro-oligosaccharide products generated from agarose digestion by beta-agarase and $\mathrm{HCl}$ in liquid chromatography systems. Carbohydrate research. 2008;343(14):2443-50. doi: 10.1016/j.carres.2008.06.019. PubMed PMID: 18701090 .

43. Kazlowski B, Pan CL, Ko YT. Monitoring and preparation of neoagaro- and agaro-oligosaccharide products by high performance anion exchange chromatography systems. Carbohydrate polymers. 2015;122:351-8. doi: 10.1016/j.carbpol.2014.09.003. PubMed PMID: 25817679.

44. Han JP, Huang YY, Ye J, Xiao MT. Screening and identification of a bacterium capable of converting agar to neoagaro oligosaccharides. Acta Microbiologica Sinica. 2015;55(9):1126-32. 
45. Fan JM, Xie CQ, Jia J, Yin XU, Zhang CX. Opitimization of extraction processing on polysaccharides from Pleurotus eryngii by cellulase enzymolysis. Food Science and Technology. 2013;38:192-6. doi: 10.13684/j.cnki.spkj.2013.03.004.

46. Liu JB, Zhao SN, Lin SY, Zhang Y, Ren M. Process optimization on extracting collagen with enzymolysis method from black fungus. Transactions of the Chinese Society of Agricultural Engineering. 2012;28:282-6. doi: 10.3969/j.issn.1002-6819.2012.13.045.

47. Hong SJ, Lee JH, Kim EJ, Yang HJ, Park JS, Hong SK. Toxicological evaluation of neoagarooligosaccharides prepared by enzymatic hydrolysis of agar. Regulatory Toxicology and Pharmacology. 2017;90:9-21. doi: 10.1016/j.yrtph.2017.08.001. PubMed PMID: 28782575.

48. Mei J, Shao J, Wang Q, Wang H, Yi Y, Ying G. Separation and quantification of neoagaro-oligosaccharides. Journal of Food Science and Technology-mysore. 2013;50(6):1217-21. doi: 10.1007/s13197-011-0448-3. PubMed PMID: 24426038; PubMed Central PMCID: PMC3791241.

\section{Supporting information}

S1 Fig. The ESIMS spectra of NA2, NA6, NA8, NA10 and NA12

S2 Fig. ${ }^{1} \mathrm{H}$ NMR spectra of NA2, NA6, NA8, NA10 and NA12

S3 Fig. ${ }^{13}$ C-NMR spectra of NA2, NA6, NA8, NA10 and NA12 


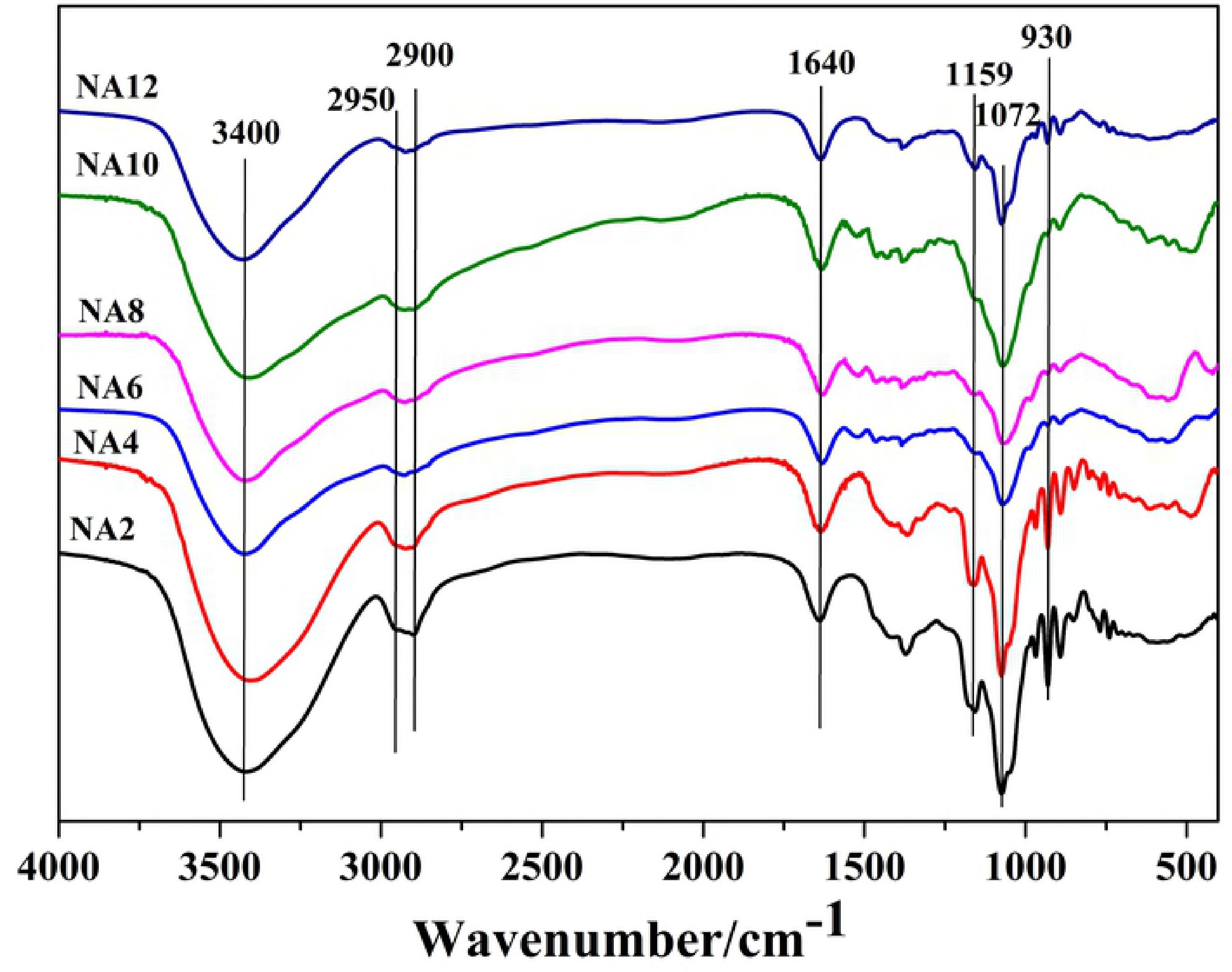




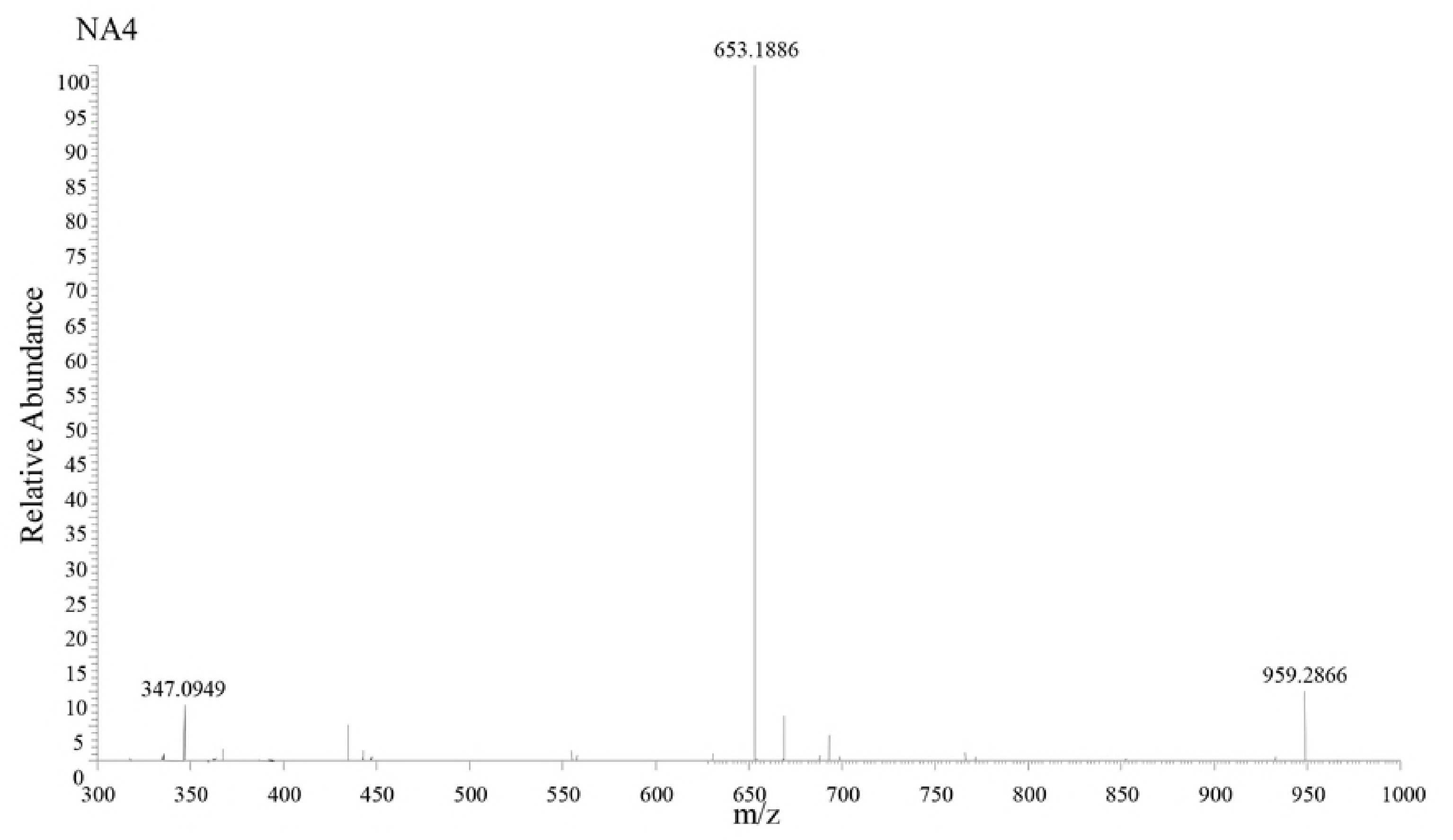




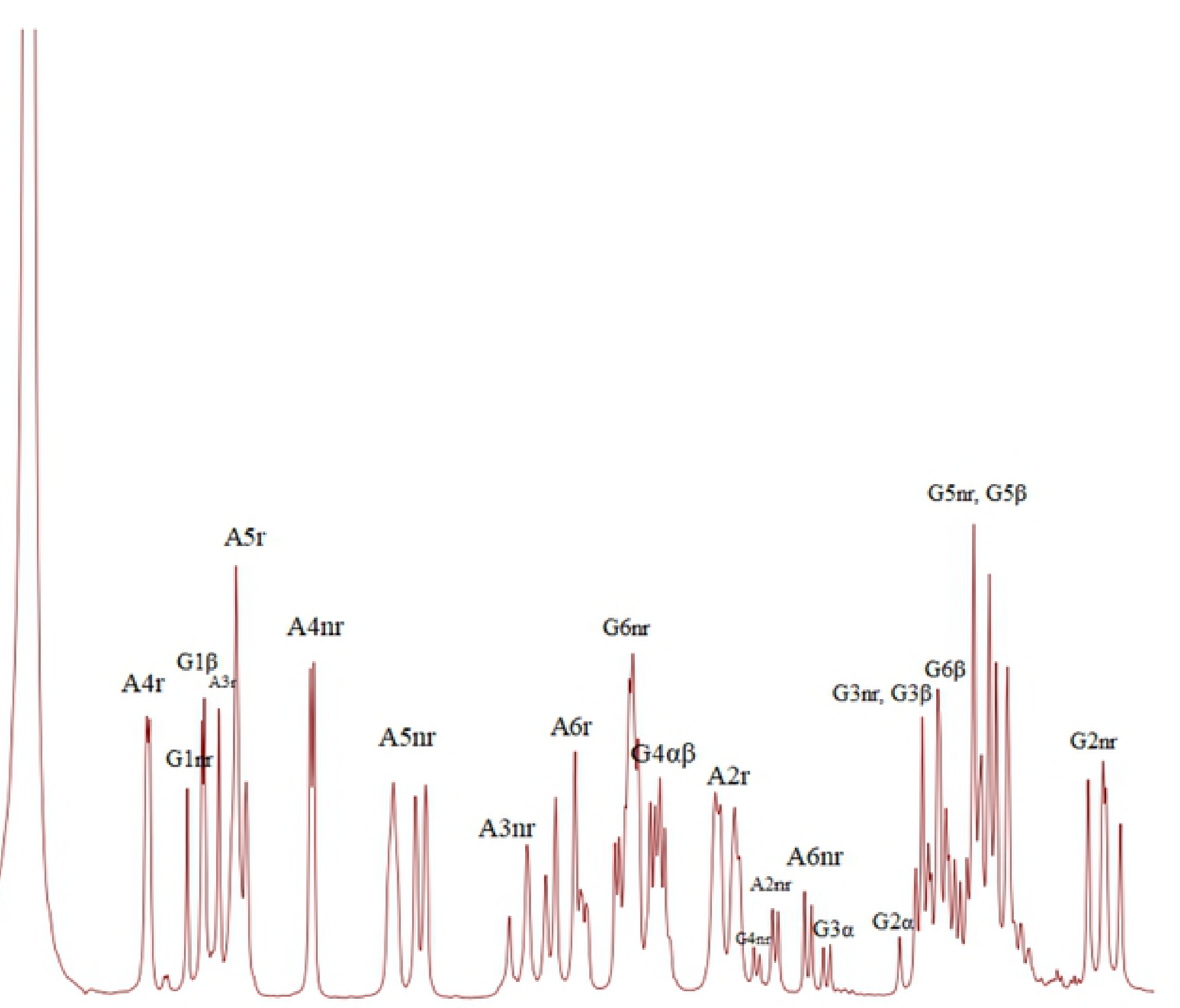

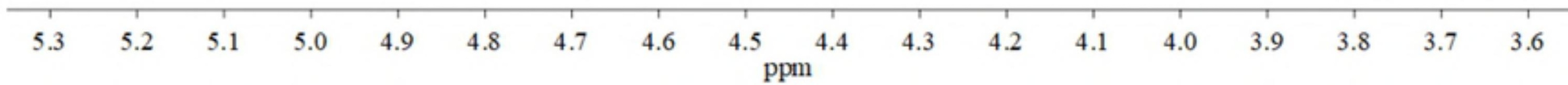




\section{$\mathrm{Anr}+\mathrm{A}+\mathrm{Ar}$}

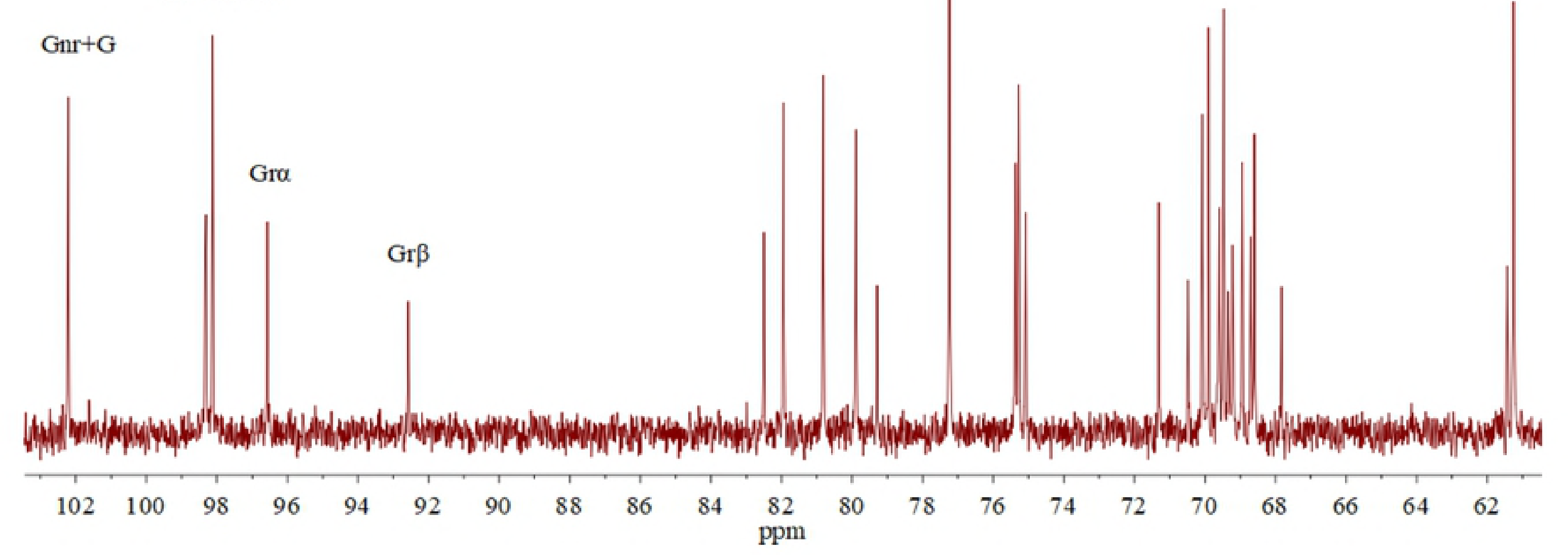



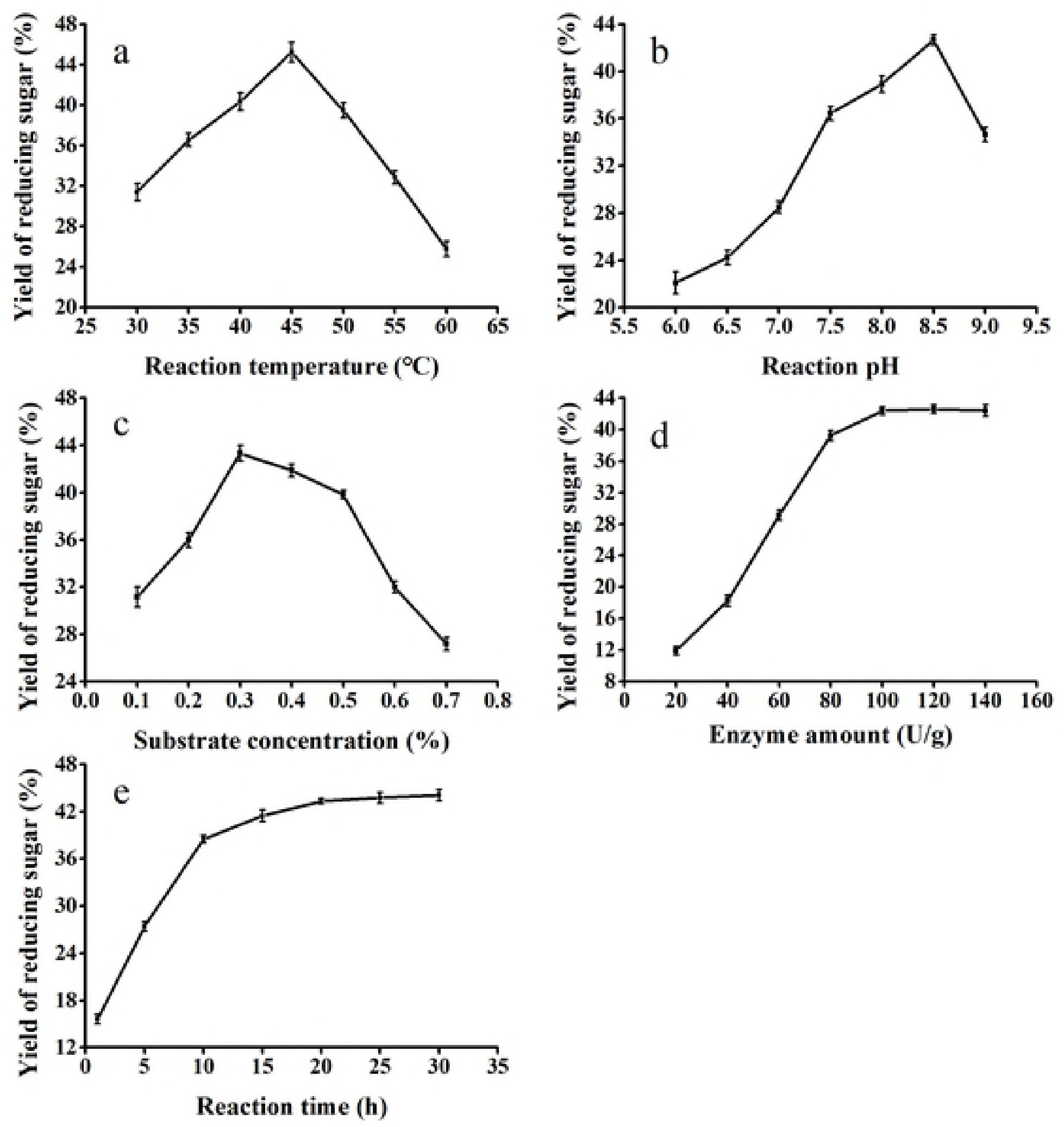


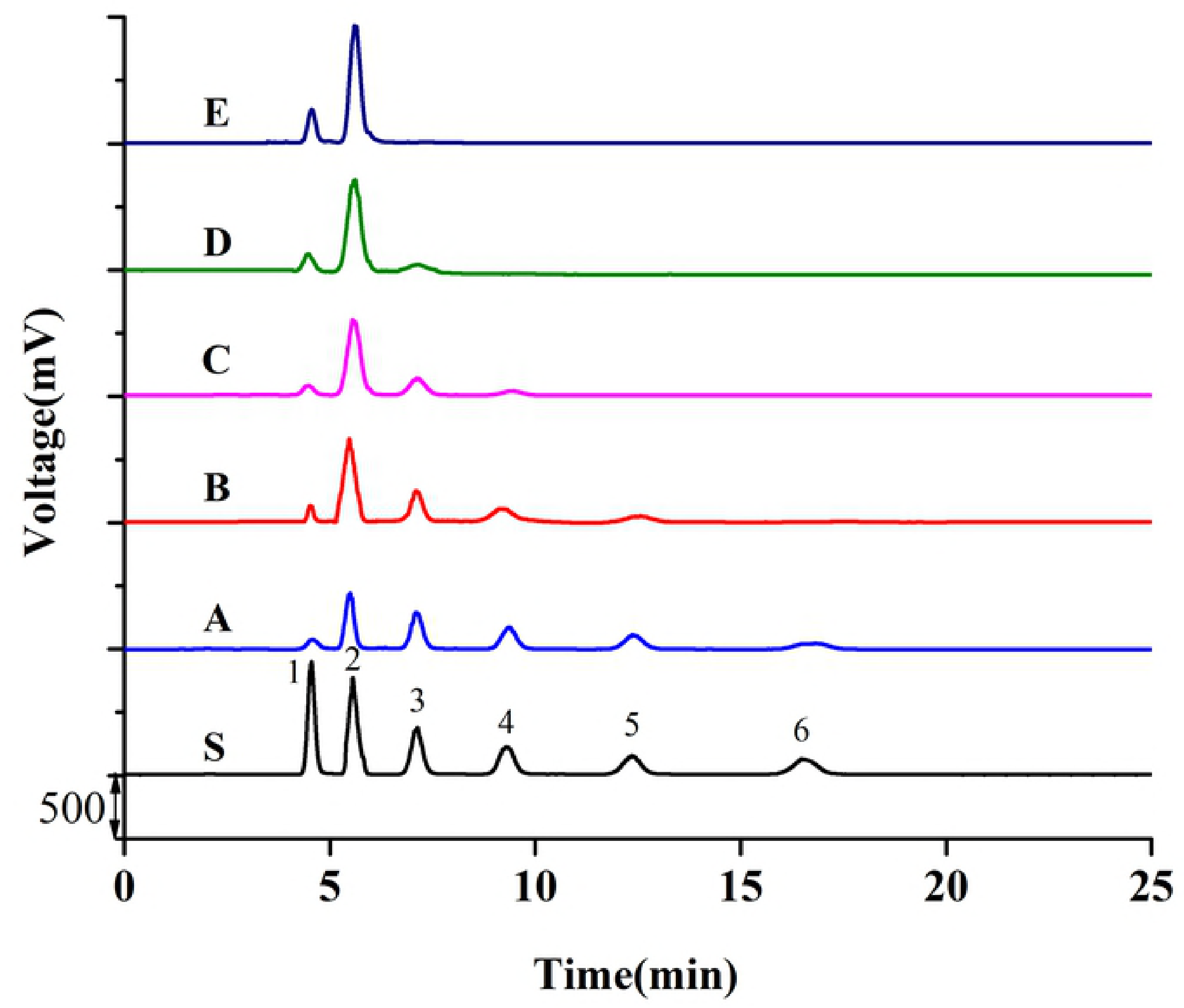




\section{$\mathrm{NA} 2-$}

NA4 -

NA6 -

NA8 -

NA10 -

NA12 - 


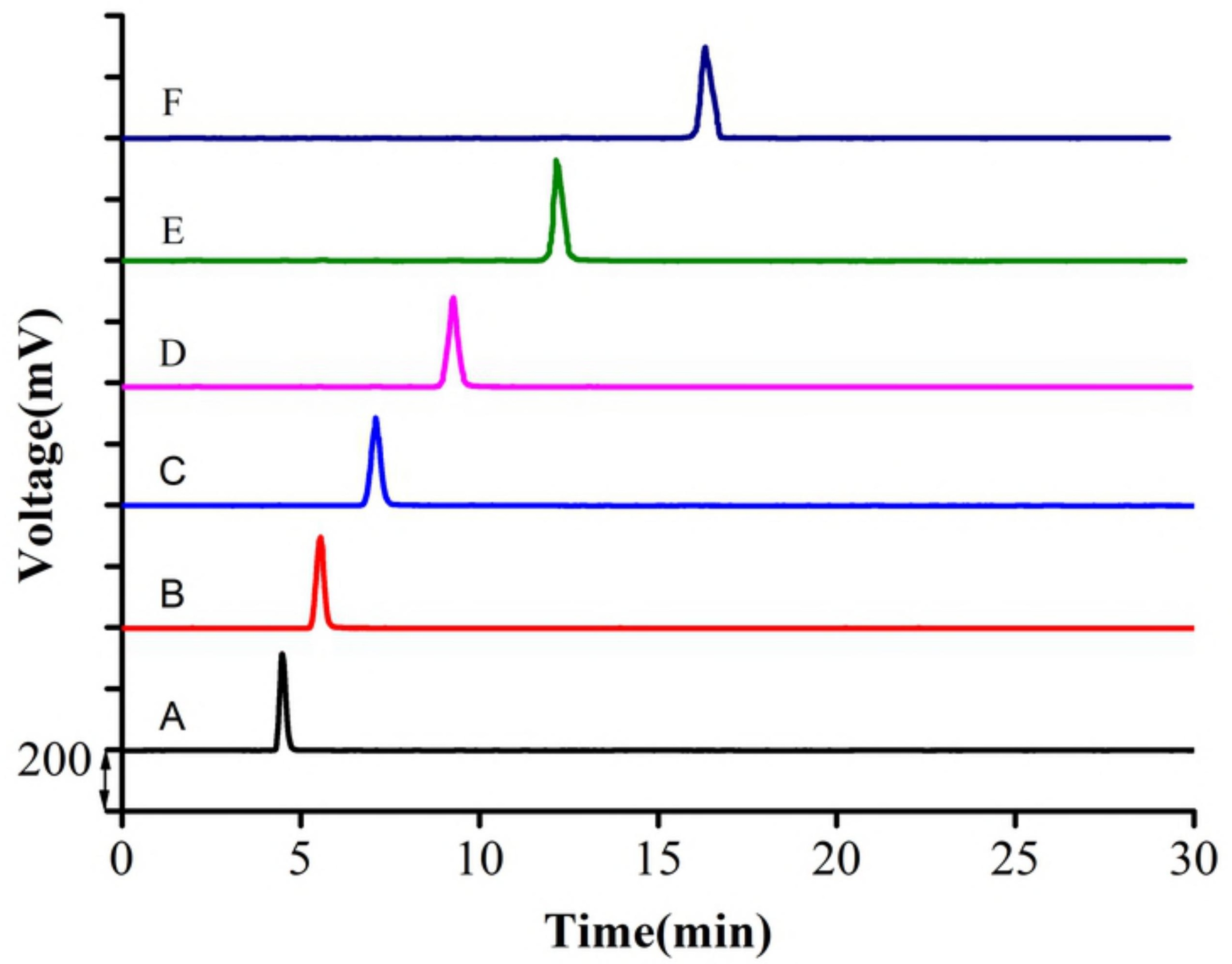

